\title{
Forensic Analysis of Hair by Scanning Electron Microscopy in Domesticated and Wild animals
}

\author{
O.P. Choudhary ${ }^{1^{*}}$ and Priyanka ${ }^{2}$ \\ ${ }^{1}$ Department of Veterinary Anatomy and Histology, College of Veterinary Sciences and \\ Animal Husbandry, Central Agricultural University (I), Selesih, Aizawl-796015, \\ Mizoram, India \\ ${ }^{2}$ Department of Veterinary Microbiology, College of Veterinary Sciences and Animal \\ Husbandry, Central Agricultural University (I), Selesih, Aizawl-796015, Mizoram, India \\ *Corresponding author
}

\section{A B S T R A C T}

\section{Keywords}

Forensic, Hair, Domestic, Wild animals, SEM

Article Info

Accepted:

10 January 2019

Available Online:

10 February 2019
Hair identification is a complex and important aspect in view of forensic investigation. The use of scanning electron microscopy in wildlife forensic cases has been described for species identification in this review article. The surface cuticular pattern, cross section and medullary index provides the information regarding the domestic and wild species of the animals. Elemental analysis through EDS provides significant information of the hair sample of specific sample which can further be used as a geographical region and species identification tool. The elements present inside the hair can be tested for both intra and inter animal differences using element percentage and atomic percentage analysis like Sulfur (S), Iron (Fe), Potassium (K) and Calcium (Ca).

\section{Introduction}

India is referred to be one of the richest biodiverse nations of the world. India is home for 400 mammalian species out of which 129 species are protected under Wildlife (Protection) Act 1972 (India) and a arbitrator to Convention on International Trade in endangered species. Illegal trade of the wildlife products are key risk to conservation and responsible for regional obliteration to these species. In forensic identification, the studies on animal hair has remain limited to cross-species comparisons from the same family, but there is also considerable changes within family like scale layer differentiation growth pattern, tensile strength, granulation and elemental profile of the animal hair. The early pioneering work on species characterizations from hair includes studies by McCurtie (1886), Hausman (1920, 1944), and Hardy and Plitt (1940). Descriptive guides on microscopic hair characteristics for some important mammalian species of particular regions have been contributed by Brunner and Coman (1974), Moore et al., 
(1974),

(1991).

\section{Collection of samples}

The hairs samples from various regions of animal body can be taken using forceps with gloves to avoid contamination and packed in zip lock bags followed by paper envelops. These hair samples can also be collected from near to poaching area, from surroundings of dead animals, live animals etc.

\section{Processing of the hair samples}

The collected hair samples are then to be washed with absolute alcohol at room temperature for about 24 hours followed by packing in fresh zip lock bags and stored at $4^{\circ} \mathrm{C}$ till further analysis. The hair can also be washed with the help to shampoo to remove the excess dirt conditions like dandruff. For Scanning electron microscopy hair samples can be dissected in pieces of $5 \mathrm{~mm}$ size leaving $3 \mathrm{~mm}$ from root side with the help of stereomicroscope. The dissected hair samples placed on the carbon sample holder. The mounted samples on the sample holder coated with gold by using auto fine coater and observed under a Scanning Electron Microscope. The photographs used in the present paper are original and captured by the authored during his $\mathrm{PhD}$ period at GB Pant University of Agriculture and Technology, Pantnagar, Uttarakhand

The samples can now be tested with the help of SEM for measurements such as scale count, scale structure, scale height, hair shape, hair diameter and surface damage, whether physical or chemical etc. at variable magnifications. Elemental analysis of hairs can be done by using Energy Dispersive Spectrum (EDS) coupled with SEM for analysis of different elements of periodic table.

\section{General structure of hair}

Hairs are mainly composed of three different layered regions- cuticle, cortex and medulla (Figure 1 and 2). Outer layer cuticle shows scales arranged like tiles which is differentiated in two parts, (a) inner endocuticle with a pitted honeycombed structure with ridges which opposes digestion by trypsin, and (b) outer exocuticle which is smooth, featureless and tryptic digestable, along with these two layers a chemically inert epicuticle is also located around the scales (Hicks, 1977). The main constituent of the hair is cortex composed of cigar shaped cells which varies in size according to keratin type (Houck and Budowle, 2002).

Perhaps the best way to explain the structure of hair is to compare it with a lead or graphite pencil, where the innermost lead or graphite layer represents the medulla, the wooden portion is the cortex, and the outermost paint layer is the thin layer of cuticle (Choudhary et al., 2014).

\section{Hair as forensic identification}

The information on hair development, growth, and chemical components and research projects on hair of Domesticated and Wild animals fills volumes. The short article to follow will certainly not be able to cover all the information on hair, but will, perhaps, give a little insight into the characteristics of hair and the role SEM can play in bringing these characteristics into focus. Histological studies have shown certain structural relationships of hairs to be usually predictable, though not invariable. The normally existing correlations are cuticular scales, medullae, cortical cells and pigment patterns of the hair.

Since growth is managed by cells arising from a mass of rapidly proliferating tissue near the 
base of the hair follicles, hair growth patterns show up on the surface scale patterns. These cells grow by differentiating into a core of keratinized material composed of two interlocked parts-the hair proper and its internal root sheaths. During growth, the core passes upward through the wall of the follicle, the external root sheath. Root sheaths and dermal papilla are important in determining hair shape, size, and movement. Several glands and skin cells are involved (which will not be delved into, since the above description of hair growth is not the prime concern, but shows some of the things involved in influencing hair structure besides hormones, vitamins, gland functions, environment and genetic factors).

Nutritive and traumatic factors can modify normal rhythm of hair growth. Thyroid hormones have a physiologically stimulating action on hair growth. The lack of adrenocorticotrophic hormones (ACTH), disease, injury or environmental stress causes a depression on growth of hair. Of the eighteen orders of Domesticated and Wild animals, all grow differently structured hair, (Figures 1 and 2) which reveals either the outer structure, or the size, or the shape of the hair differently in each case. Some animals grow hair in wave patterns; others grow hair continuously, as in sheep and humans.

In addition to growth mechanisms previously described, there are other individual and diagnostic pathological, chemical and abnormal conditions which affect surface characteristics of hair, and may be used as criteria for identifying hair of animal's / individuals.

The variability of animal's hair in each race is greater than the variability of hairs on a single individual's head. The hair of the dog is most nearly circular in cross-sections; coarser and straighter hair lends itself to a more circular shape. Goat hair is the flattened being quite curly in nature, as tends to be the case with flattened ovaloid cross-sectioned hair. The elephant hair sections are intermediate in cross-section shape.

Stereo photographs can be taken on the SEM simply by taking one picture of the sample at one angle, then, tilting the sample an additional $2^{\circ}$ to $10^{\circ}$ to another angle, and taking another picture, it is the angle at 'which your eyes would normally see the sample, since all depth is seen by the brain interpreting two images from two angles in relation to the two eyes.

The stereo image can be seen by focusing at a distance, holding the picture about 10 inches from the eyes, and viewing the stereo pair. Stereo glasses era also be used to view the image. Stereo photographs show the great depth of field, not achieved with light optical instruments since the depth of field would not be the same and resolution would be very poor with the optical instruments in general use. With the stereo means, scaly structure and surface features not readily noticed or determined before literally pop into view. This, "too, is a great advantage in hair identification, image can be seen by focusing at a distance, holding the picture about 10 inches from the eyes, and viewing the stereo pair. Stereo glasses era also be used to view the image.

Stereo photographs show the great depth of field, not achieved with light optical instruments since the depth of field would not be the same and resolution would be very poor with the optical instruments in general use. Elemental analysis using SEM-EDS have revealed about the percentage of Sodium, Sulfur, Calcium and Potassium on the basis of weight $\%$ and Atomic \% respectively. 
Fig.1 Absolute alcohol for washing of collected samples

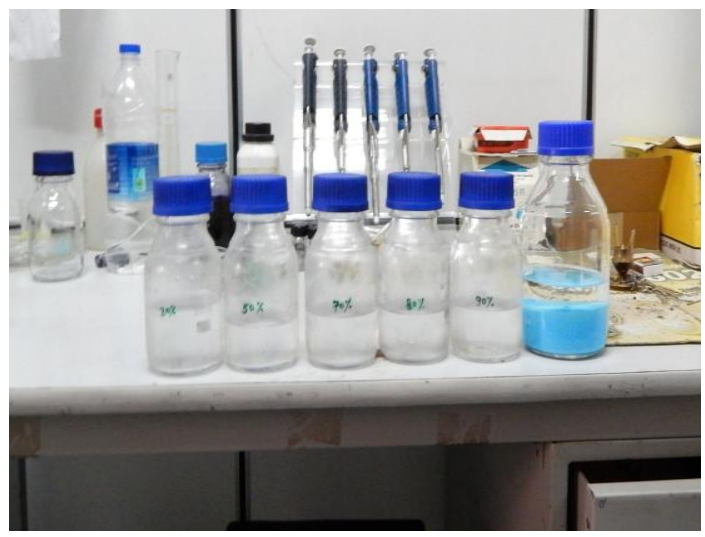

Fig.2 SEM grid with carbon tape for mounting of the dissected hair

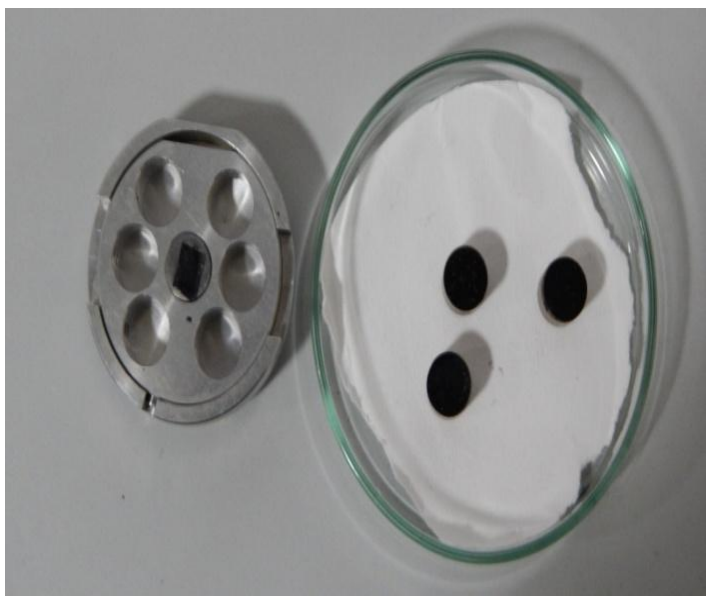

Fig.3 JEOL gold Coater for gold coating of the hair samples

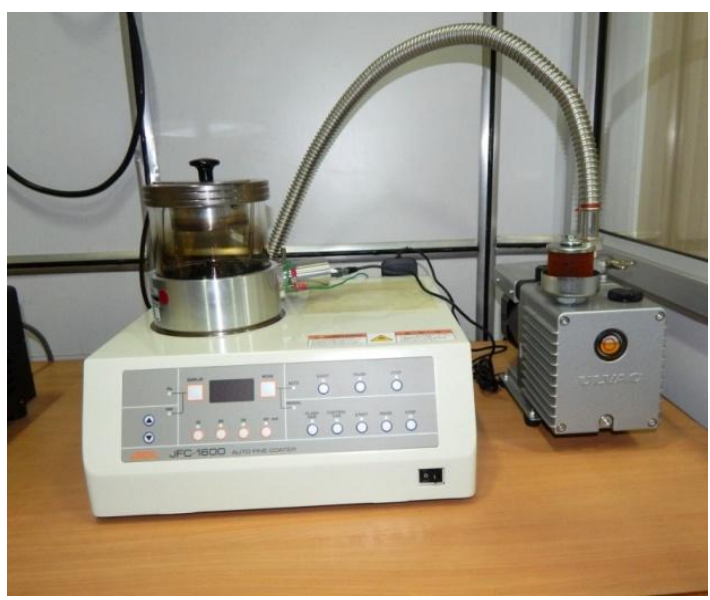


Fig.4 JEOL-SEM for EDS analysis of the hair processed hair samples

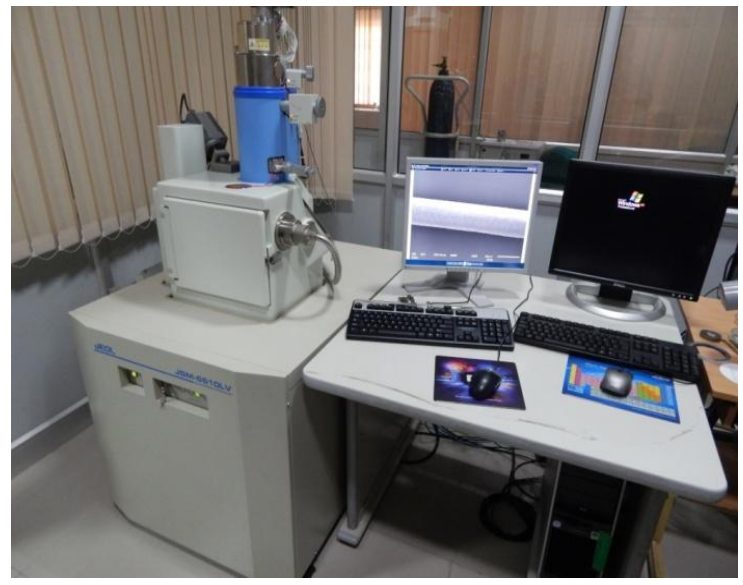

Fig.5 Scanning electron micrograph showing inner medulla and outer cortex part of hair of dog

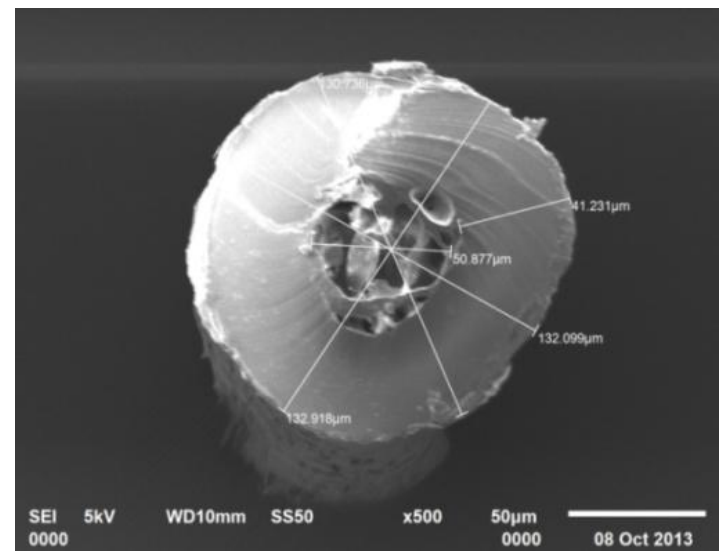

Fig.6 Scanning electron micrograph showing outermost layer of overlapping and transparent scales of hair of dog

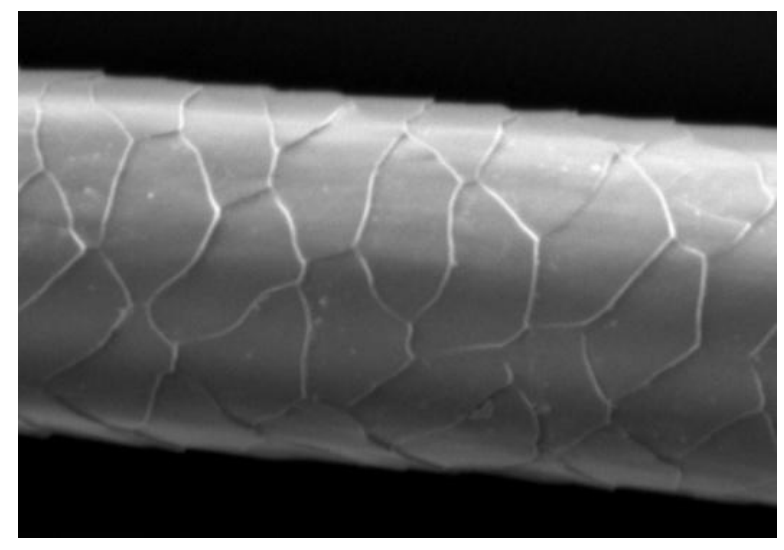


Fig.7 Distribution curve of different elements in the hair of dog

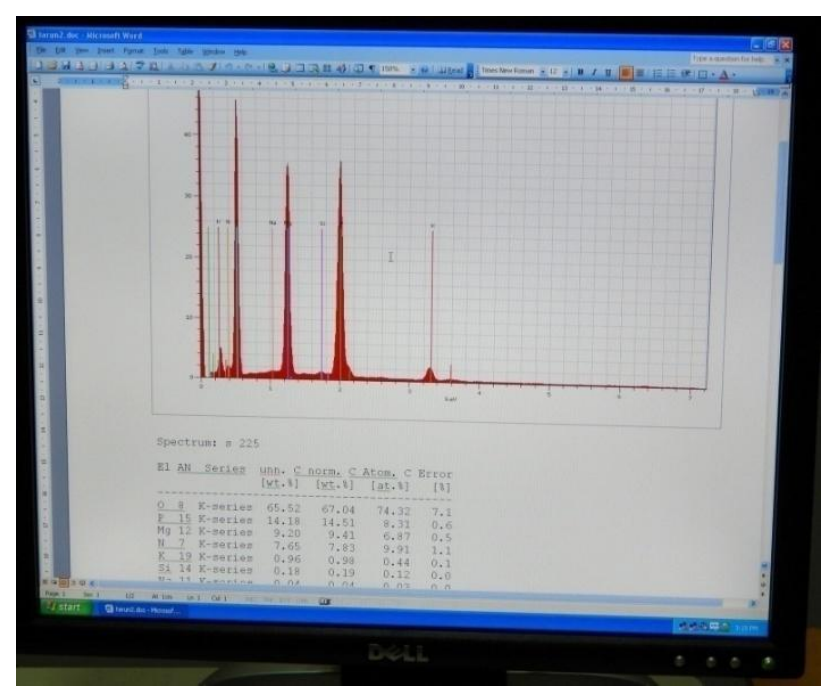

Hair identification is a complex and important aspect in view of forensic investigation. It entails many tests and uncertainties still exist in drawing conclusions to determine whether a single hair is identifiable from a certain individual. The Scanning Electron Microscope alone may not be able to cause final conclusions to be drawn, as however, with farther tests and comparative studies, this type of study certainly will eventually lead to a more positive identification of the hairs, since it definitely shows improvement over the optical means of identification regarding structural, surface morphology, coupled with other scientific data. The use of scanning electron microscopy in wildlife forensic cases has been described for species identification. The surface cuticular pattern, cross section and medullary index provides the information regarding the species. Researchers have revealed the scale architecture of regular mosaic with smooth margins of shahtoosh wool. But when the hair evidence from different species from the same family were found to be blended together than the investigation becomes quite tough and typical so the present study which incorporates the scale layer difference between closely related species can provide the information regarding its species to the forensic expert. Elemental analysis through EDS provides significant information of the hair sample of specific sample which can further be used as a geographical region and species identification tool (Dahiya et al., 2013). The elements present inside the hair were tested for both intra and inter animal differences using element percentage and atomic percentage analysis like Sulfur (S), Iron (Fe), Potassium $(\mathrm{K})$ and Calcium $(\mathrm{Ca})$.

The major conclusion of work is that it provides a vital information regarding identification of three different species from the Felidae family on the bases of their scale layer differentiation pattern whereas it was quite difficult to identify them on the bases of previously reported literature and provides a new area for the identification of hairs.

\section{Future aspects of work}

Although there is a vast range of literature available regarding medullary index, scale pattern, pigmentation and growth there is a need to develop the database of the differentiation in scale layers which does not only provide the information regarding species but also give information regarding his disease history as well as environmental 
exposure with the help of techniques like SEM, EDS etc. This study will work as a tool/primary source in forming the database of the scale layer pattern for the identification of closely related species from the same family where the examiner faces problems regarding identification.

\section{References}

Appleyard HM. 1978. Guide to Identification of Animal Fibres. 2 $2^{\text {nd }}$ Edn., Wool Industries Research Association, Leeds.

Choudhary OP, Dhote BS, Bharti SK and Sathapathy S. 2014. The Advantages of the Scanning Electron Microscope in the forensic Studies of Hair in Domesticated and Wild animals. Souvenir and Abstract, XXVIII Annual Convention of Indian Association of Veterinary Anatomists, pp: 153.

Brunner $\mathrm{H}$ and Coman BJ. 1974. The Identification of Mammalian Hair. Inkata Press, Melbourne.

Dahiya MS and Yadav SK. 2013. Scanning Electron Microscopic Characterization and Elemental Analysis of Hair: A Tool in Identification of Felidae
Animals. Journal of Forensic Research 4: 178.

Hardy JI and Plitt TM 1940. An improved method for revealing the surface structure of fur fibers. U.S. Dept. Interior Wildlife Circ. 7: 10

Hausman LA. 1920. Structural characteristics of the hair of mammals. Am. Nat. 54: 496-523.

Hausman LA. 1944. Applied microscopy of hair. Scient. Monthly. 59: 195-202

Moore T.D, Spence LE and Dugnolle EE. 1974. Identification of the Dorsal Guard Hairs of Some Mammals of Wyoming. Game and Fish Dept. Wyoming.

Teerink BJ. 1991. Atlas and Identification Key on Hair of West-European Mammals. Cambridge University Press.

Hicks JW. 1977. Microscopy of Hairs: A Practical Guide and Manual. Federal Bureau of Investigation, U.S. Government Printing Office, Washington DC.

Houck MM, Budowle B. 2002. Correlation of microscopic and mitochondrial DNA hair comparisons. Journal of Forensic Science 47: 964-967.

\section{How to cite this article:}

Choudhary, O.P. and Priyanka. 2019. Forensic Analysis of Hair by Scanning Electron Microscopy in Domesticated and Wild animals. Int.J.Curr.Microbiol.App.Sci. 8(02): 10281034. doi: https://doi.org/10.20546/ijcmas.2019.802.120 